\title{
Does a lower self-concept contribute to mental health disparities of diverse immigrant youth from middle childhood to late adolescence?
}

\author{
J. Busch ${ }^{1^{*}}$ (D) C. Claus ${ }^{2+}$, S. Schneider ${ }^{2}$ and R. G. Siefen ${ }^{3}$
}

\begin{abstract}
Background: Three out of ten children in Germany have immigrant backgrounds and this proportion is expected to further increase in subsequent years. While immigrant youth have been found more vulnerable to developing symptoms of depression and anxiety, the underlying mechanisms of how such disparities unfold during youth development are still understudied. Some previous research has found that immigrant youth are at risk of experiencing a less positive self-concept compared to non-immigrant youth. We investigated whether the self-concept mediates mental health disparities and explored variability in such associations from middle childhood to late adolescence.
\end{abstract}

Methods: Overall 1839 children and adolescents aged $6-21$ years $(M=14.05$ years, $S D=3.03,49.8 \%$ female, $n=782$ with immigrant status) participated in a cross-sectional self-report survey in classroom settings using scales from the Beck Youth Inventories II (Beck et al. in Beck Youth Inventories - Second Edition, Psychological Corporation, San Antonio, 2005) to assess self-concept and symptoms of depression and anxiety. Links between immigrant status, age, self-concept and symptom levels of depression as well as anxiety were examined using hierarchical regression and moderated mediation models.

Results: Immigrant youth reported higher symptom levels of depression and anxiety than their non-immigrant peers but did not differ in their self-concepts. Hypothesized moderated mediation models were not fully supported and self-concept neither mediated the link between immigrant status and depression nor immigrant status and anxiety. However, self-concept was a significant predictor for symptom levels of depression as well as anxiety, with stronger associations in adolescents.

Conclusions: Our study substantiates previous findings that immigrant youth in Germany have overall increased symptom levels of depression and anxiety compared to non-immigrant youth. Our study however does not support that immigrant youth have a more negative self-concept and that the self-concept mediates such internalizing mental health disparities. Findings match previous evidence that developing a positive attitude towards the self is linked to better mental health. Beyond that, our findings suggest that mental health interventions addressing the

\footnotetext{
*Correspondence: julian.busch@rub.de

${ }^{\dagger}$ J. Busch and C. Claus have share first authorship

${ }^{1}$ Faculty of Psychology, Department of Child and Family Research, Ruhr-

University, Bochum, Germany

Full list of author information is available at the end of the article
} 
self-concept could be especially relevant when targeting adolescents. Further research is needed to deepen the understanding of the mediating processes between migration status and mental health variables.

Keywords: Self-concept, Depression, Anxiety, Immigrant, School, Germany

\section{Background}

About one-fifth of all children and adolescents in Germany experience mental health problems $[2,3]$ with symptoms of depression and anxiety being among the most common in these age groups [4]. Evidence is alarming, as developing a clinically relevant anxiety or depressive disorder during childhood and adolescence impairs the unfolding of developmental potentials and increases the risk for mental health problems during adulthood [2]. Notably, a higher risk for developing such common internalizing mental health problems has been reported for diverse groups of children and adolescents from immigrant families residing in European countries [5, 6] including newly arrived refugees [7] and those, whose families have lived in the resettlement country for the second or even third generation [8]. Symptoms associated with depression and anxiety have been consistently linked to severe psychological and social consequences among immigrant youth; Plener et al. [9] found a higher prevalence of suicide attempts compared to their nonimmigrant counterparts, van Oort et al. [10] substantiated links to the emergence of socio-economic disparities between immigrant and non-immigrant populations during adulthood.

With a growing number of children and adolescents in Germany, currently 36\%, have an immigrant status [11], research on the foundations of systematic differences in the mental health status between immigrant and non-immigrant youth is becoming increasingly relevant. Specifically, a better understanding of distinctive mechanisms is an important step to mitigate mental health disparities within the diverse societies of tomorrow. A literature review including twenty global studies on immigrant youth in resettlement countries [12] suggested a lower socio-economic status as a mediator for increased levels of mental health problems among young immigrant populations. However, the reviewed studies also supported that the relations between holding an immigrant background and experiencing mental health problems often persisted above and beyond the explanatory effects of socio-economic status [12]. Some of the reviewed evidence demonstrated within-family stress between first- and second-generation immigrants to additionally account for mental health problems in immigrant youth. Comparing the reviewed studies on withinfamily conflicts between first- and second-generation immigrants yielded that such findings could be at least partially confounded with age [13]. When immigrant youth are compared to non-immigrant youth of the same age group disparities seem to diminish [14]. Withinfamily stress is thus more likely related to a specific developmental period and the specific effects linked to immigrant background still remain to be delineated further. Previous findings on the mediating processes were moreover critically discussed as studies were conducted in different and also heterogenous populations, applied varying methodology and, even partially, yielded inconsistent evidence. For example, both lower and higher levels of mental health problems in immigrant youth when compared to non-immigrant youth were found in some studies $[15,16]$. One methodological critique addressed the absence of univocal definitions of key constructs such as "immigrant background" or the measurements for mental health problems in immigrant youth [12].

Another recent literature review distinctively studied potential mechanisms and discussed pathways of how mental health disparities between immigrant and nonimmigrant populations could unfold [17]. Determinants such as language proficiency, sex, age and, again, socioeconomic status were found to be linked to differences in health literacy among immigrant and non-immigrant populations that subsequently are linked to mental health disparities. Authors of that review additionally discussed how heightened access barriers within healthcare systems disadvantage immigrant populations (i.e., less health care benefits/ less opportunities to develop health literacy) and thus likely contribute to mental health disparities [17]. Other studies adopting ecocultural perspectives found cultural distance to the host community [18, 19], social stress [6] and acculturative stress [20] to be linked to increased levels of especially internalizing mental health problems among immigrant youth.

Previous studies on the determinants and mechanisms of mental health disparities in immigrant youth so far have less focused on the question of how those disparities unfold within-person and have seldom considered differences across developmental periods. To shed light on such genuine developmental psychopathological processes, this study investigates the selfconcept as a correlate for internalizing mental health problems during youth development. Self-concept is defined as the overall system of beliefs, cognitive and affective attitudes towards oneself. The term summarizes a person's self-evaluation regarding specific 
aspects such as one's academic abilities, physical performance or self-regulation capacities [21, 22]. The selfconcept constitutes intrinsic cognitive schemata mainly determined by feedback from social environments [23]. Self-directed feedback is processed intra-personally in order to form a coherent picture of oneself. Previous research [24] has described two strategies of how the self-concept can be structurally organized, namely compartmentalization and integration. Compartmentalization describes a self-image that is structured in different categories containing either positive or negative components of oneself, while integration forms a self-concept that consists of positive as well as negative information about the self [24]. Evidence that links the structure of the self-concept to self-esteem suggests that individuals who tend to compartmentalize their self-concept may experience difficulties in accessing coherent information about oneself, leading to an overall more negative evaluation of the self [25].

A negative and unstable self-concept has also been linked to symptoms of anxiety and depression among children and adolescents in general populations [26-30]. For example, a self-report survey with children aged 9 to 13 years in school settings showed that those with symptoms of depression and anxiety also reported lower levels of global self-esteem and a lower quality of life. Such links were stronger in older children of the sample [31]. However, no study has yet focused on the self-concept's distinctive significance for internalizing mental health problems among immigrant youth. Some previous evidence supports the notion that immigrant children and adolescents are more vulnerable to a lower self-concept [32] - although such evidence is still limited and remains inconsistent. Conceptually, immigrant status is a critical biographical characteristic for children and adolescents as it is linked to intra- and interindividual processes of adaptation and to an increased risk of experiencing stigmatization [33]. Even for those immigrant children and adolescents without personal migration experiences, their origin culture as conveyed by relatives and the migration experience of parents or grandparents still forms the children's and adolescents' self-ascriptions [33, 34]. Socio-cultural discontinuities that immigrant youth likely experience between the family context and the host country's society can impede the formation of a positive and integrative self-concept throughout youth development $[35,38,39]$. While immigrant children and adolescents are exposed to the host countries' influences in school contexts and peer-networks, they are often taught the language, traditions and values of their origin country at home [36]. Such potentially opposing contexts can result in the development of a fragmented "self-culture" whose principles are ambiguous, context-dependent, conflicting and which can be accompanied by symptoms of withdrawal and self-insecurity $[8,37]$. The fragmentation could thus promote a compartmentalized structure of the self-concept.

Adding a developmental perspective, an inner focus on the emerging self-concept arises with the beginning of puberty and peaks during adolescence [38]. Adolescence marks a hypersensitive period to all kinds of social stimuli when a coherent self-concept is formed via information seeking [40, 41]. During that period, schools provide a central social context to establish a coherent self-concept. Since immigrant youth are at heightened risk to experience cultural discontinuities between school and non-school contexts, forming a coherent and positive self-concept in schools could be particularly challenging for this group [42, 43]. Reviewing the research on immigrant children and adolescents from different generations in school-contexts, previous work has mainly focused on the academic components of the self-concept. For example, Mitchell [44] overall found a lower academic self-concept for immigrant adolescents with Central American backgrounds attending schools in the United States and substantiated links to lower levels of well-being. The author, however, did not find differences regarding the generational status of immigration (i.e., first-, second- and third-generation). In further studies a lower self-concept was correspondingly considered a socially-determined and multi-directional pathogenic influence $[45,46]$, also impacting internalizing mental health problems [47]. It is therefore necessary to extend research on the self-concept among immigrant youth to self-directed cognitions and emotions within school settings.

Investigating the role of the self-concept throughout youth development, our study goal was to better understand the foundations of symptoms of depression and anxiety in immigrant children and adolescents. Specifically, our study hypotheses were to replicate previous findings that $(\mathrm{H} 1)$ immigrant children and adolescents experience higher symptom levels of depression and anxiety than non-immigrants in school settings, and (H2) that a lower self-concept was linked to those increased symptom levels. Based on this replication, we expected (H3) the self-concept to mediate the respective links between immigrant status and depression as well as anxiety, and ( $\mathrm{H} 4)$ that the effects on symptom levels are stronger during adolescence. The theoretical model reflecting the study hypotheses is illustrated in Fig. 1a.

\section{Methods}

\section{Study design and sample}

Overall, $N=1907$ children and adolescents participated in a cross-sectional self-report survey that we conducted 


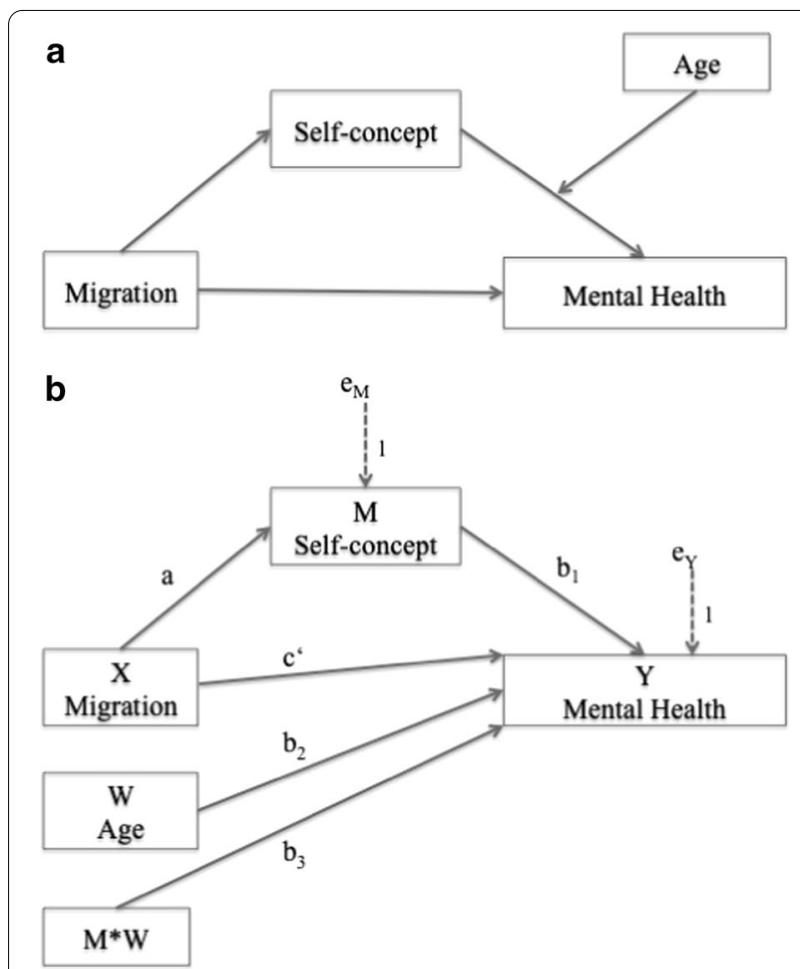

Fig. 1 Panel a illustrates the moderated mediation model according to our study hypotheses. We proposed that immigrant youth report increased symptom levels of depression and anxiety $(\mathrm{H} 1)$, lower self-concept predicts higher symptom levels $(\mathrm{H} 2)$, self-concept mediates the link between immigrant status and symptom levels ( $\mathrm{H} 3)$ and that age moderates the hypothesized mediation (H4). Panel b illustrates the statistical realization as suggested by Hayes [56] and corresponds to the analyses reported in Table 6

in primary and secondary schools across Germany's most populous and multicultural federal state, North-Rhine Westphalia. We chose schools in different regions (i.e., varying the degree of urbanization) and neighbourhoods and considered all types of German secondary schools to balance the student sample. At study enrollment, teachers informed parents using written handouts in several languages. Parents who agreed to letting their children participate in the study signed an informed consent. All students in primary and secondary school classrooms were eligible to participate if they were regular members of the class, had a minimum of second grade reading comprehension and presented their parental declaration of consent. We intended to classify our full study sample post-hoc into a non-immigrant and an immigrant group based on their own or familial history of migration. Data were collected via classroom surveys according to a standardized administration protocol. No more than 8 participants per study facilitator were assessed at once in secondary schools. For primary school children, group surveys with a maximum of 4 children per study facilitator were conducted to increase levels of assistance, when necessary. Due to difficulties in the recruitment of primary schools, additional data collections were conducted in sports and social clubs for younger children following the same procedure. All study facilitators were advanced undergraduate or graduate students in psychology, supervised by clinically experienced researchers.

\section{Measures}

The study questionnaire comprised a demographic section including participants' age, sex, migration status and the indicators for parents' socio-economic status. The highest number of parental school years and parents' profession according to the International Socio-Economic Index of Occupational Status [48] were used to assess the familial socio-economic status of the sample. To measure participants' internalizing mental health problems, subscales of the German version of the Beck Youth Inventories-II (BYI-II) were used [49]. The BYI-II is a set of overall 5 self-report questionnaires with 20 items each for assessing depression, anxiety, self-concept, anger and disruptive behaviour in children and adolescents aged 7-18 years. Depending on children's age and reading competence, each questionnaire takes about 8-10 min for completion. Participants responded on a 4-point Likert scale (0, "never" to 3 , "always") to rate how frequently each statement applied to their daily cognitions, emotions and behaviours during the past two weeks. The BYI-II is designed for a large age range, as it operationalizes symptoms in easy language. That also facilitates the use of the BYI-II for immigrant groups, whose German language skills are often on average lower. In accordance with our study aims, the BYI-II questionnaires on depression, anxiety and self-concept were used in this study. We rigorously adhered to the BYI-II manual for scoring and missing value handling.

\section{Self-concept}

The positively worded self-concept inventory (Beck Self-Concept Inventory for Youth, BSCI-Y) assesses cognitive and emotional perceptions of competency and self-worth. It comprises the overall system of selfdirected perceptions, cognitive and affective attitudes toward one's own person such as the perceived social role (e.g., "People want to be with me"), the personal strengths (e.g., "I am good at telling jokes") and self-acceptance (e.g., " I like myself "). The inventory showed good internal consistency (Cronbach's $\alpha$ for immigrants $=0.86$; Cronbach's $\alpha$ for non-immigrants $=0.86$ ).

\section{Depression}

The depression inventory (Beck Depression Inventory for Youth, BDI-Y) screens for symptoms of depression 
Table 1 Socio-demographic characteristics of the sample

\begin{tabular}{|c|c|c|}
\hline Variable & Immigrant ( $n=782$ ) & $\begin{array}{l}\text { Non-immigrant } \\
(n=1057)\end{array}$ \\
\hline Sex (female, \%) & 47.4 & 51.5 \\
\hline Age in years, mean (SD) & $14.08(2.99)$ & $14.03(3.06)$ \\
\hline \multicolumn{3}{|l|}{ Parental SES (\%) } \\
\hline Highest (14-19 years) & 23.5 & 30.4 \\
\hline High (12-13 years) & 23.1 & 30.4 \\
\hline Medium (10-11 years) & 35.5 & 34.1 \\
\hline Low (up to 9 years) & 17.9 & 5.1 \\
\hline \multicolumn{3}{|l|}{ ISCED School Type (\%) } \\
\hline ISCED-1 (Primary School) & 44.0 & 32.6 \\
\hline ISCED-2 (Lower Secondary) & 31.8 & 19.1 \\
\hline ISCED-3 (Upper Secondary) & 24.2 & 48.3 \\
\hline \multicolumn{3}{|l|}{ Migration status, n (\%) } \\
\hline First Generation & $130(16.6)$ & - \\
\hline Second Generation & $614(78.5)$ & - \\
\hline Third Generation & $38(4.9)$ & - \\
\hline
\end{tabular}

SES, Parental socio-economic status was defined by parents' highest number of schooling years. The migration status refers to the child or adolescent reporting a history of immigration. For ISCED School Type, we classified schools that youth of our sample attended according to the "International Standard Classification of Education" [74]

in line with the established criteria of the Diagnostic and Statistical Manual of Mental Health Disorders, Fourth Edition [50]. This included low mood (e.g., "I feel sad"), somatic complaints (e.g., "I have trouble sleeping") and latent suicidal tendencies (e.g., "I wish I were dead"). The inventory showed excellent internal consistency (Cronbach's $\alpha$ for immigrants $=0.93$; Cronbach's $\alpha$ for non-immigrants $=0.93$ ).

\section{Anxiety}

The anxiety inventory (Beck Anxiety Inventory for Youth, BAI-Y) screens for core symptoms of anxiety disorders relevant to children and adolescents such as general anxiety disorder, panic disorder and social phobia. In line with the Diagnostic and Statistical Manual of Mental Health Disorders, Fourth Edition [49], items reflect participants' specific fears including in different situations (e.g., "I worry when I am at school"), worried social cognitions (e.g., "I worry people might get mad at me") and physiological symptoms (e.g., "I get shaky"). The inventory showed very good internal consistency (Cronbach's $\alpha$ for immigrants $=0.89$; Cronbach's $\alpha$ for non-immigrants $=0.91$ )

\section{Data analyses}

We performed data preparations and moderation models using SPSS version 25 [51] and regression models using $\mathrm{R}$ Version 3.5.0 [52]. Alpha-error probability was $p<0.05$ (two-tailed) in all analyses. We adjusted for multiple comparisons using the "Multiple Comparisons
Calculator" [53] in accordance with the procedure established by Bonferroni and Holm [54]. All $p$-values, considered in hypothesis testing are displayed in bold in the study tables. We indicate significance only for those $p$-values after adjustment but consistently report unadjusted $p$-values throughout all tables for coherence in the illustration. For hypotheses on mediation, we interpret bootstrap confidence intervals (CIs). In preliminary analyses, we inspected data and checked statistical assumptions for parametric statistical procedures and computed a correlation matrix on variables considered in main analyses.

\section{Hypotheses 1 and 2}

For H1, we compared levels of depression, anxiety and self-concept between immigrant and non-immigrant youth while controlling for the covariates age, sex and parental socio-economic status. We therefore calculated two-step hierarchical regression models and, additionally, calculated $R^{2}$ to determine the effect sizes. For $\mathrm{H} 2$, we similarly examined the impact of the self-concept on depression and anxiety, also controlling for age, sex and parental socio-economic status.

\section{Hypotheses 3 and 4}

We used second stage moderated mediation models [55] to test $\mathrm{H} 3$ and $\mathrm{H} 4$ as theoretically illustrated in Fig. 1a. We performed modeling using PROCESS, third version, a macro for SPSS developed by Hayes [56] that 
Table 2 Descriptive analysis of the Beck Youth Inventories (second edition) for symptoms of depression and anxiety by migration status and age

\begin{tabular}{|c|c|c|c|c|c|c|}
\hline $\begin{array}{l}\text { Study group per } \\
\text { measure }\end{array}$ & M & Min & Max & SD & Med & Clin \\
\hline \multicolumn{7}{|l|}{ Depression } \\
\hline \multicolumn{7}{|l|}{ Immigrants } \\
\hline Children & 10.13 & 0 & 60 & 10.56 & 7 & 11.51 \\
\hline Adolescents & 9.60 & 0 & 52 & 8.90 & 8 & 8.95 \\
\hline \multicolumn{7}{|l|}{ Non-Immigrants } \\
\hline Children & 8.30 & 0 & 56 & 8.51 & 6 & 8.80 \\
\hline Adolescents & 8.75 & 0 & 47 & 8.97 & 6 & 7.57 \\
\hline \multicolumn{7}{|l|}{ Anxiety } \\
\hline \multicolumn{7}{|l|}{ Immigrants } \\
\hline Children & 15.99 & 0 & 60 & 10.51 & 15 & 6.27 \\
\hline Adolescents & 14.90 & 0 & 41 & 8.51 & 14 & 8.70 \\
\hline \multicolumn{7}{|l|}{ Non-Immigrants } \\
\hline Children & 12.81 & 0 & 60 & 9.00 & 12 & 8.99 \\
\hline Adolescents & 13.42 & 0 & 56 & 8.97 & 11 & 6.15 \\
\hline \multicolumn{7}{|l|}{ Self-Concept } \\
\hline \multicolumn{7}{|l|}{ Immigrants } \\
\hline Children & 38.86 & 4 & 60 & 9.45 & 40 & 5.88 \\
\hline Adolescents & 38.38 & 10 & 59 & 7.68 & 39 & 7.93 \\
\hline \multicolumn{7}{|l|}{ Non-Immigrants } \\
\hline Children & 39.47 & 12 & 60 & 9.09 & 40 & 8.80 \\
\hline Adolescents & 37.89 & 9 & 60 & 7.63 & 38 & 6.43 \\
\hline
\end{tabular}

$M$, mean scores. SD, standard deviation. Med, Median. Four-point Likert scale from 0 ("never") to 4 ("always"). Clin, Participants above clinical cut-off (T-Score of 60 and above) in percent. Children are between 6.92 and 13.92 years, adolescents are between 14.05 and 21.17 years of age. The full sample consisted of $n=357$ children and $n=425$ adolescents with immigrant backgrounds, and $n=445$ children and $n=612$ adolescents without immigrant backgrounds

follows a regression-based approach to integrate mediation and moderation in a conditional process analysis [56]. As displayed in Fig. 1b, and contrary to the widely used historical method by Baron and Kenny [57], the regression-based approach quantifies the indirect effect between the antecedent $X$, the mediator $M$ and the consequent $Y$ itself and does not require an association between $X$ and $Y$ as a precondition [56]. The second stage moderated mediation model uses bootstrapping $(k=5000)$ to obtain estimates for $95 \%$ bias-corrected CIs for the conditional indirect effects in moderated mediation. The indirect effect is calculated for different conditional values of the moderator variable (i.e., two age groups-youth below or above 14 years of age). Conditional indirect effects are statistically significant when zero is not included between the lower and upper bound of the $95 \%$ bias-corrected bootstrap confidence intervals generated for different values of the moderator variable $W$. The bootstrapping method is also superior to the Sobel test [58] due to higher statistical power and independency of the normal distribution of the underlying data [56].

\section{Results}

\section{Descriptive analysis of the study sample}

Of the $N=1907$ children and adolescents, two did not state their age, another 66 did not complete the demographic section on the immigrant status of their parents or grandparents and were therefore excluded a-priori. This resulted in an effective study sample of $N=1839$. About half of the participants were female $(n=49.8 \%)$ and participants were on average 14.05 years old $(S D=3.03)$. A large proportion of the sample comprised immigrant children and adolescents $(n=782,42.5 \%)$ with most of these being in the second immigrant generation. Immigrant youth themselves or their families originated from 69 different countries with Turkey $(n=194,10.5 \%)$, Poland $(n=111,6.0 \%)$, and Russia $(n=42,2.3 \%)$ being the most frequently stated countries. Immigrant youth overall came from families with a lower socio-economic status compared to non-immigrant youth. Given the population-based data collection procedure and demographic characteristics of the obtained study population, 
Table 3 Correlation matrix of depression, anxiety, self-concept, age, sex, SES and migration status

\begin{tabular}{|c|c|c|c|c|c|c|c|}
\hline Variable & Depression & Anxiety & Self-concept & Age & Sex & SES & Migration \\
\hline Depression & 1 & & & & & & \\
\hline Anxiety & 0.697 & 1 & & & & & \\
\hline Self-Concept & -0.456 & -0.283 & 1 & & & & \\
\hline Age & 0.022 & -0.003 & -0.144 & 1 & & & \\
\hline Sex & -0.189 & -0.196 & 0.128 & 0.025 & 1 & & \\
\hline SES & -0.082 & -0.100 & 0.112 & -0.074 & 0.095 & 1 & \\
\hline Migration & -0.069 & -0.116 & 0.000 & -0.008 & -0.04 & 0.217 & 1 \\
\hline
\end{tabular}

SES, parental highest socio-economic status. Dichotomous variables (Sex, Migration) compared to metric variables using point-biserial correlation. Categorical Variable (SES, 4 groups) compared to metric variables using biserial correlation. Dichotomous and categorical variables compared to each other using Cramer's $V$

the immigrant group in our sample reflects the general population with immigrant backgrounds in Germany [47]. The measurements used in this study were created for an age range of 7-18 years, reflecting the schooling period in Germany. Seventy-six participants were outside of the defined age range, that is, 8 participants younger than 7 years and 68 participants older than 18 years. Since these students were members of the classrooms and their exclusion did not yield different results, they remained part of the presented analyses. Note that our effective study sample includes missing values of less than 0.9 percent per analyzed symptom variable. Due to the overall large sample size, we decided to conduct analyses based on that data. Table 1 reports basic socio-demographic information of the effective study sample. Table 2 gives an overview on the symptoms of depression and anxiety and the self-concept of the effective study sample. Table 3 shows correlations between study measures and socio-demographic information.

\section{Examination of Hypotheses 1 and 2}

In analyses for $\mathrm{H} 1$, the covariates sex and parental socio-economic status predicted symptom levels of depression and anxiety while in regressing self-concept the covariate age likely had additional predictive value. As expected in $\mathrm{H} 1$, immigrant children and adolescents reported increased symptom levels of depression $\left(B_{\text {Unstandardized }}=1.223\right.$, adjusted $\left.p<0.05\right)$ and anxiety ( $B_{\text {Unstandardized }}=2.179$, adjusted $p<0.01$ ) but, contrary to our expectations, did not report a lower self-concept compared to non-immigrants $\left(B_{\text {Unstandardized }}=0.195\right.$, adjusted $\left.p>0.05\right)$. Beyond the covariates, the migration status explained small additional proportions of unique variance in symptoms of anxiety $\left(\Delta R^{2}=0.013\right)$ and depression $\left(\Delta R^{2}=0.004\right.$; see Table 4). In analysis for $\mathrm{H} 2$, we found the self-concept to influence symptoms of depression such that a lower self-concept was linked to increased symptom levels
$\left(B_{\text {Unstandardized }}=-0.477\right.$, adjusted $\left.p<0.001\right)$. Consistently, self-concept also predicted increased levels of anxiety in the same direction $\left(B_{\text {Unstandardized }}=-0.283\right.$, adjusted $p<0.001)$. While the influence of the selfconcept on both outcomes was substantial, it was larger on symptom levels of depression $\left(\Delta R^{2}=0.183\right)$ than of anxiety $\left(\Delta R^{2}=0.063\right)$. Details of the stepwise hierarchical regression analyses on $\mathrm{H} 2$ are presented in Table 5.

\section{Moderated mediation analyses with the outcome depression (Hypotheses 3 and 4)}

We predicted that age would moderate the indirect effect of the migration status on depression via self-concept (see Fig. 1a). The migration status did not influence the self-concept (path $a$ ). We consistently did not substantiate an overall indirect effect based on bootstrap confidence intervals (i.e., reduction of predictive value from path c to path c'). More specifically, we found no indirect effects of the focal predictor migration status on symptoms of depression for both age groups (children: indirect effect $=-0.016,95 \% \mathrm{CI}:-0.358,0.350$; adolescents: indirect effect $=-0.020,95 \%$ CI: $-0.485,0.439)$. Selfconcept thus did not represent a mediator for the demonstrated link between migration status and symptoms of depression. These findings contradicted the expectations of H3. However, the effect of self-concept on symptoms of depression depended on age as evidenced by the statistically significant interaction between $M$ and $W\left(\right.$ path $\left.b_{3}\right)$. While there was a negative link between self-concept and symptoms of depression in both age groups, this effect was stronger in the older group (children: $\beta=-0.450$, $p<0.001$; adolescents: $\beta=-0.587, p<0.001)$. These findings were in line with $\mathrm{H} 4$ as a lower self-concept had a higher impact on increased symptom levels of depression during adolescence (i.e., participants older than 14 years of age). For further details see Table 6. 
Table 4 Hierarchical regression analysis for migration status predicting symptoms of depression, anxiety and self-concept

\begin{tabular}{|c|c|c|c|c|c|c|c|c|}
\hline \multirow[t]{2}{*}{ Variable } & \multicolumn{4}{|l|}{ Model 1} & \multicolumn{4}{|l|}{ Model 2} \\
\hline & $B$ & $S E$ & $t$ & $p$ & $B$ & $S E$ & $t$ & $p$ \\
\hline \multicolumn{9}{|l|}{ Depression } \\
\hline Intercept & 27.732 & 1.236 & 22.440 & $<.001$ & 26.815 & 1.277 & 21.003 & $<.001$ \\
\hline Age & 0.066 & 0.070 & 0.932 & .352 & 0.067 & 0.070 & 0.957 & .339 \\
\hline Sex & 3.624 & 0.426 & 8.503 & $<.001$ & 3.666 & 0.426 & 8.612 & $<.001$ \\
\hline SES-medium & -0.936 & 0.750 & -1.247 & .213 & -0.593 & 0.759 & -0.781 & .435 \\
\hline SES-high & -1.983 & 0.772 & -2.569 & .010 & -1.548 & 0.786 & -1.969 & .049 \\
\hline SES-highest & -2.432 & 0.772 & -3.149 & .002 & -2.003 & 0.786 & -2.549 & .011 \\
\hline Migration & & & & & 1.223 & 0.439 & 2.787 & $.025^{*}$ \\
\hline$\Delta R^{2}$ & \multicolumn{4}{|c|}{$R^{2}=.046, F(5,1800)=17.27, p<.001$} & \multicolumn{4}{|c|}{$\Delta R^{2}=.004, F(1,1799)=7.765, p=.005$} \\
\hline \multicolumn{9}{|l|}{ Anxiety } \\
\hline Intercept & 33.395 & 1.246 & 26.805 & $<.001$ & 31.761 & 1.281 & 24.792 & $<.001$ \\
\hline Age & -0.010 & 0.071 & -0.139 & .890 & -0.007 & 0.070 & -0.097 & .923 \\
\hline Sex & 3.846 & 0.430 & 8.951 & $<.001$ & 3.921 & 0.427 & 9.178 & $<.001$ \\
\hline SES-medium & -0.438 & 0.756 & -0.579 & .563 & 0.173 & 0.761 & 0.227 & .820 \\
\hline SES-high & -1.679 & 0.778 & -2.158 & .031 & -0.904 & 0.789 & -1.146 & .252 \\
\hline SES-highest & -2.638 & 0.778 & -3.390 & $<.001$ & -1.875 & 0.789 & -2.378 & .018 \\
\hline Migration & & & & & 2.179 & 0.441 & 4.947 & $.007^{*}$ \\
\hline$\Delta R^{2}$ & \multicolumn{4}{|c|}{$R^{2}=.053, F(5,1800)=20.01, p<.001$} & \multicolumn{4}{|c|}{$\Delta R^{2}=.013, F(1,1799)=24.468, p<.001$} \\
\hline \multicolumn{9}{|l|}{ Self-concept } \\
\hline Intercept & 63.598 & 1.136 & 55.988 & $<.001$ & 63.452 & 1.176 & 53.960 & $<.001$ \\
\hline Age & -0.340 & 0.065 & -6.113 & $<.001$ & -0.395 & 0.065 & -6.107 & $<.001$ \\
\hline Sex & -2.337 & 0.392 & -5.965 & $<.001$ & -2.330 & 0.392 & -5.943 & $<.001$ \\
\hline SES-medium & 0.812 & 0.689 & 1.177 & .239 & 0.866 & 0.699 & 1.239 & .215 \\
\hline SES-high & 1.751 & 0.709 & 2.469 & .014 & 1.820 & 0.724 & 2.514 & .012 \\
\hline SES-highest & 2.695 & 0.710 & 3.797 & $<.001$ & 2.762 & 0.724 & 3.817 & $<.001$ \\
\hline Migration & & & & & 0.195 & 0.404 & 0.481 & $.630^{n s}$ \\
\hline$\Delta R^{2}$ & \multicolumn{4}{|c|}{$R^{2}=.046, F(5,1800)=17.27, p<.001$} & \multicolumn{4}{|c|}{$\Delta R^{2}=.0001, F(1,1799)=0.2318, p=.630$} \\
\hline
\end{tabular}

$B$, unstandardized regression coefficient. $S E$, standard error of $B$. Sex, $1=$ female, $0=$ male. SES, parental socio-economic status. Migration status: $1=$ immigrant background, $0=$ no immigrant background. Uncorrected $p$-values for estimates reflecting study hypotheses given in bold, alpha-error corrected thresholds applied to indicate significance at ${ }^{* * *} p>.001,{ }^{* *} p>.01,{ }^{*} p>.05, n s=$ not significant

\section{Moderated mediation analyses with the outcome anxiety (Hypotheses 3 and 4)}

We predicted that age would moderate the indirect effect of migration status on symptoms of anxiety via self-concept (see Fig. 1a). The migration status did not affect the self-concept (path $a$ ). The indirect effect as estimated by bootstrap confidence intervals did not yield an effect. Specifically, we found no indirect influence of the focal predictor migration status on symptoms of anxiety for both age groups (children: indirect effect $=-0.009,95 \%$ CI: $-0.198,0.193$; adolescents: indirect effect $=-0.411$, 95\% CI: $-0.345,0.307)$. Self-concept thus did not function as a mediator for the demonstrated link between migration status and symptoms of anxiety. These findings similarly contradicted the expectations of $\mathrm{H} 3$. However, the effect of self-concept on symptoms of anxiety depended on age, as evidenced by the statistically significant interaction between $M$ and $W$ (path $b_{3}$ ). While there was an overall negative link between selfconcept and symptoms of anxiety, this link was consistently stronger in the older group (children: $\beta=-0.242$, $p<0.001$; adolescents: $\beta=-0.411, p<0.001)$. These findings were in line with $\mathrm{H} 4$ as a lower self-concept had a higher impact on increased symptom levels of anxiety during adolescence. Overall, the moderated mediation model explained more variance of the outcome variable depression $\left(R^{2}=0.229\right)$ than of the outcome variable anxiety $\left(R^{2}=0.103\right)$. For further details see Table 6 .

\section{Discussion}

This study integrated perspectives of developmental science and clinical child psychology to examine the selfconcept as an intra-individual correlate of mental health disparities between immigrant and non-immigrant youth 
Table 5 Hierarchical regression analysis for self-concept predicting symptoms of depression and anxiety

\begin{tabular}{|c|c|c|c|c|c|c|c|c|}
\hline \multirow[t]{2}{*}{ Variable } & \multicolumn{4}{|l|}{ Model 1} & \multicolumn{4}{|l|}{ Model 2} \\
\hline & $B$ & $S E$ & $t$ & $p$ & $B$ & $S E$ & $t$ & $p$ \\
\hline \multicolumn{9}{|l|}{ Depression } \\
\hline Intercept & 27.732 & 1.236 & 22.440 & $<.001$ & 58.053 & 1.840 & 31.553 & $<.001$ \\
\hline Age & 0.066 & 0.070 & 0.932 & .352 & -0.123 & 0.064 & -1.923 & .055 \\
\hline Sex & 3.624 & 0.426 & 8.503 & $<.001$ & 2.510 & 0.387 & 6.486 & $<.001$ \\
\hline SES-medium & -0.936 & 0.750 & -1.247 & .213 & -0.549 & 0.675 & -0.813 & .416 \\
\hline SES-high & -1.983 & 0.772 & -2.569 & .010 & -1.148 & 0.695 & -1.652 & .099 \\
\hline SES-highest & -2.432 & 0.772 & -3.149 & .002 & -1.147 & 0.697 & -1.646 & .100 \\
\hline Self-concept & & & & & -0.477 & 0.023 & -20.677 & $<.001^{* * *}$ \\
\hline$\Delta R^{2}$ & \multicolumn{4}{|c|}{$R^{2}=.046, F(5,1800)=17.27, p<.001$} & \multicolumn{4}{|c|}{$\Delta R^{2}=.183, F(1,1799)=427.53, p<.001$} \\
\hline \multicolumn{9}{|l|}{ Anxiety } \\
\hline Intercept & 33.395 & 1.246 & 26.805 & $<.001$ & 51.399 & 1.994 & 25.784 & $<.001$ \\
\hline Age & -0.010 & 0.071 & -0.139 & .890 & -0.122 & 0.069 & -1.758 & .079 \\
\hline Sex & 3.846 & 0.430 & 8.951 & $<.001$ & 3.185 & 0.419 & 7.594 & $<.001$ \\
\hline SES-medium & -0.438 & 0.756 & -0.579 & .563 & -0.210 & 0.731 & -0.285 & .776 \\
\hline SES-high & -1.679 & 0.778 & -2.158 & .031 & -1.183 & 0.753 & -1.571 & .116 \\
\hline SES-highest & -2.638 & 0.778 & -3.390 & $<.001$ & -1.876 & 0.755 & -2.484 & .013 \\
\hline Self-concept & & & & & -0.283 & 0.025 & -11.332 & $<.001^{* * *}$ \\
\hline$\Delta R^{2}$ & \multicolumn{4}{|c|}{$R^{2}=.053, F(5,1800)=20.01, p<.001$} & \multicolumn{4}{|c|}{$\Delta R^{2}=.063, F(1,1799)=128.4, p<.001$} \\
\hline
\end{tabular}

$B$, unstandardized regression coefficient. SE, standard error of $B$. Sex, $1=$ female, $0=$ male. $t, t$-value. $p, p$-values for $t$-tests on regression coefficient and intercept. SES, parental socio-economic status. $R^{2}$ and $F$-tests for differences to intercept-only models. Uncorrected $p$-values for estimates reflecting study hypotheses in bold, alphaerror corrected thresholds applied to indicate significance at ${ }^{* * *} p>.001,{ }^{* *} p>.01,{ }^{*} p>.05$

throughout youth development. We could support that immigrant youth report more symptoms of depression and anxiety than non-immigrant youth and could also show that a lower self-concept is linked to such symptoms. However, our analyses did not yield self-concept as a significant mediator for these associations. Despite this null effect we found that age moderated the association between self-concept and internalizing mental health problems. We discuss study evidence along the study hypotheses.

\section{Immigrant children and adolescents experience more} symptoms of depression and anxiety than non-immigrants We hypothesized that having a migration background would link to increased levels of internalizing mental health problems. Indeed, migration status predicted symptoms of depression as well as anxiety. These findings contribute evidence to the ongoing debate whether migration status is linked to an increased risk for mental health problems (as hypothesized in this study), or alternatively facilitates processes of resilience. Contrary to our study propositions, some evidence on adolescent students in several European and other high-income countries substantiated the "immigrant (health) paradox", that is, immigrant youth have a better mental health [59-62]. Based on a large dataset, our findings are however more consistent with our initial expectations of linking the migration status to lower mental health. Our evidence is supported by a stream of literature on the "mental health gap" between immigrant and non-immigrant youth [63, 64]. Possible explanations for the opposing propositions in the literature open two pathways. First, while many of the previous studies focus on immigrants from specific origin countries, our sample was more likely drawn from general immigrant populations in Germany including a variety of countries, ethno-cultural origins as well as immigrant generations. Through collecting data in non-exclusive classroom interviews, we did not limit our immigrant sample to specific groups. Our study sample thus more likely reflects the current and diverse population of children and adolescents with immigrant status in Germany. Some previous evidence suggests that the "immigrant health paradox" could encompass first-generation immigrants only [65]. Second, as our data was collected in school contexts, the identified mental health disparities might reflect consequences of social and ethnocultural discontinuities that immigrant children and 
Table 6 Model coefficients for the moderated mediation models predicting symptoms of depression or anxiety

\begin{tabular}{|c|c|c|c|c|c|c|c|c|}
\hline \multirow[t]{3}{*}{ Antecedent } & \multicolumn{8}{|c|}{ Consequent } \\
\hline & \multicolumn{4}{|c|}{$M$ (Self-concept) } & \multicolumn{4}{|c|}{$Y$ (Depression) } \\
\hline & Path & Coeff & $S E$ & $p$ & Path & Coeff & $S E$ & $p$ \\
\hline $\begin{array}{l}X \text { (Migration } \\
\text { status) }\end{array}$ & $a$ & .035 & .399 & .931 & $c^{\prime}$ & 1.350 & .384 & $<.001$ \\
\hline $\begin{array}{l}M \text { (Self-con- } \\
\text { cept) }\end{array}$ & - & - & - & - & $b_{1}$ & -.450 & .031 & $<.001$ \\
\hline$W($ Age $)$ & - & - & - & - & $b_{2}$ & 4.767 & 1.794 & .008 \\
\hline$M \times W$ & - & - & - & - & $b_{3}$ & -.138 & .045 & $.003^{*}$ \\
\hline Constant & $i_{M}$ & 38.569 & .259 & $<.001$ & $i_{Y}$ & 26.155 & 1.268 & $<.001$ \\
\hline \multirow[t]{2}{*}{ Model } & \multicolumn{4}{|c|}{$R^{2}=-.000$} & \multicolumn{4}{|c|}{$R^{2}=.229$} \\
\hline & \multicolumn{4}{|c|}{$F(1,1814)=.001, p=.931$} & \multicolumn{4}{|c|}{$F(4,1811)=134.439, p<.001$} \\
\hline \multirow[t]{3}{*}{ Antecedent } & \multicolumn{8}{|c|}{ Consequent } \\
\hline & \multicolumn{4}{|c|}{$M$ (Self-concept) } & \multicolumn{4}{|c|}{$Y($ Anxiety) } \\
\hline & Path & Coeff & $S E$ & $p$ & Path & Coeff & $S E$ & $p$ \\
\hline $\begin{array}{l}X \text { (Migration } \\
\text { status) }\end{array}$ & $a$ & .040 & .398 & .922 & $c^{\prime}$ & 2.287 & .421 & $<.001$ \\
\hline $\begin{array}{l}M \text { (Self-con- } \\
\text { cept) }\end{array}$ & - & - & - & - & $b_{1}$ & -.242 & .034 & $<.001$ \\
\hline$W($ Age $)$ & - & - & - & - & $b_{2}$ & 6.045 & 1.963 & .002 \\
\hline$M \times W$ & - & - & - & - & $b_{3}$ & -.169 & .050 & $<.001^{* *}$ \\
\hline Constant & $i_{M}$ & 38.551 & .260 & .000 & $i_{Y}$ & 22.679 & 1.389 & $<.001$ \\
\hline \multirow[t]{2}{*}{ Model } & \multicolumn{4}{|c|}{$R^{2}=.000$} & \multicolumn{4}{|c|}{$R^{2}=.103$} \\
\hline & \multicolumn{4}{|c|}{$F(1,1820)=.001, p=.922$} & \multicolumn{4}{|c|}{$F(4,1817)=52.091, p<.001$} \\
\hline
\end{tabular}

Left column: Path $a$ for predictor $X$ (migration status) to mediator $M$ (self-concept) calculated according to Hayes [56], $R^{2}$ and $F$-tests for differences to intercept-only model. Right column: Full model paths $\left(c^{\prime}, b_{1}, b_{2}, b_{3}\right)$ in moderated mediation models with symptom scores of depression or anxiety as consequent as illustrated in Fig. 1 Panel B), adjusted $R^{2}$ and $F$-test for differences to intercept-only model. See Results section for estimates on conditional effects. $M$, mediator. $W$, moderator. $M \times W$, interaction effect. $S E$, standard error. Uncorrected $p$-values for estimates reflecting study hypotheses in bold, alpha-error corrected thresholds applied to indicate significance at ${ }^{* * *} p>.001,{ }^{* *} p>.01,{ }^{*} p>.05$

adolescents can experience between home and school contexts [32].

\section{Self-concept does not mediate the link between migration status and depression/anxiety}

We found that a lower self-concept was overall linked to increased symptom levels of depression and anxiety. This finding is in line with our expectations and previous studies on children and adolescents from general populations [28-30]. Based on the study sample, these findings moreover suggest that such associations could be generalizable to children and adolescents with diverse immigrant backgrounds.

We however did not find differences between immigrant and non-immigrant youth regarding their selfconcepts. Correspondingly, our moderated mediation models did not substantiate a lower self-concept as a mediator for the link between migration status and internalizing mental health problems. These findings contradicted our expectations of a link between migration status and self-concept. At the same time, migration status significantly accounted for higher levels of internalizing mental health problems in our sample (H2). There are two approaches to explain such patterns of expected and unexpected findings. First, more specific dimensions of the self-concept, better reflecting a compartmentalized self-structure, rather than a global, unidimensional construct, merely reflecting an integrated self-structure, could better explain links between migration status and internalizing mental health problems. As a compartmentalized structure of the self was previously linked to lower mental health status and we substantiated fewer mental health problems for immigrant youth in our study, immigrant youth could have less accurately reported on their self-concepts using this global measure. Besides, the most relevant compartments of the selfconcept that distinguish immigrant and non-immigrant youth may have not been in their cognitive foci when answering the self-concept measure. In a school context, compartments of the self-concept relating to academic components [66] likely influence cognitions when examining oneself. However, specific components of the 
self-concept, such as self-esteem or emotional stability, could have been relatively less salient for immigrant children and adolescents during study participation-even though such domains might be more strongly linked to internalizing mental health problems. Second, the selfconcept, either in global or domain-specific operationalization, is not directly associated with the migration status as we hypothesized. Considering the deductions from the coexisting immigrant paradox, immigrant youth could be better off in some health-associated psychological domains (including the self-concept), while they simultaneously show increased symptom levels in others (including symptoms of depression and anxiety).

\section{Age moderates the link between self-concept and depression/anxiety}

Consistent with our expectations, we found that adolescents' internalizing mental health problems are more strongly affected by a lower self-concept in our sample. The influence of the self-concept on the participants' mental health status was moderated by age, meaning a lower self-concept seemed to impact adolescents more strongly than children. Considering age as a proxy for youth development, our evidence highlights the importance of developmental processes for understanding the relationship between self-concept and mental health. Consistent to this model and to our evidence, a previous study found older age and more internalizing mental health problems to predict lower self-concept in a multiethnic youth group in the United States [67].

Moreover, behavioral and cognitive covariations of the self-concept with children's and adolescents' age need additional consideration when interpreting our findings. For young children, the self-concept is less validated by outside cues and tends to be more positive in general [68]. Marsh, Parada, Yeung and Healey [69] postulated that younger children defend their self-concept with "troublemaking behavior" (i.e., socially directed strategies). While our study focus was on symptoms of depression and anxiety, the specific symptoms of mental health problems with either externalizing or internalizing nuances that are associated with a low self-concept could thus vary as a function of age. Another approach to explaining age differences in the self-concept focuses on its intrapersonal organization. In a study with youth suffering from attention deficit hyperactivity disorder and internalizing mental problems [67], adolescents reported a lower self-concept than children. Study authors discussed whether such differences were either due to developmental processes influencing the organization of the self-concept or due to the stability of problem behavior which could have negatively affected the selfconcept over time. Also, in our study, continuous social withdrawal could thus have limited the reception of environmental cues that are required for establishing a stable and positive self-concept during adolescence.

\section{Strengths, limitations and future research}

There were several limitations that underline the need for future research on the identification of within-person mediators for the mental health disparities between immigrant and non-immigrant youth. First of all, the sample was collected via self-report measures in school settings. To further validate our findings and substantiate generalization, we need replication studies with more sophisticated designs, such as using multi-informant approaches and longitudinal data collections from middle childhood to late adolescence. Second, the self-concept was measured via self-report questionnaires using a unidimensional construct applicable to a variety of social settings. The use of multi-dimensional measures, for example the Self-Description Questionnaire II [45], could better assess compartmentalized structures of the self-concept. Third, the BYI-II subscales were administered to a highly heterogenous group with immigrant backgrounds. To date, constructs as operationalized in these inventories still have to prove psychometric fidelity and measurement invariance, especially with regard to respondents' ethnocultural backgrounds. Nonetheless, the present study has several strengths. It combined clinical child psychology and developmental science perspectives as it investigated the impact of the self-concept on symptoms of depression and anxiety from middle childhood to late adolescence. We included immigrant youth of the second- and third-generations from multiple origin countries to acknowledge youth's diversity in current German society, while past research on adolescent mental health has extensively focused on first-generation immigrant populations [7, 20]. Regarding the study population, our evidence is based on a regionally and socioeconomically controlled sample across the largest federal state in Germany and data was collected in a variety of different school types. However, we cannot fully preclude bias due to undetected mechanisms linked to socio-economic disparities. The parental socio-economic status of immigrant youth in our sample was on average lower in the immigrant sample. The parental socio-economic status was moreover linked to symptoms of anxiety which is consistent to previous findings.

Subsequent research needs to further investigate which within-person processes during childhood and adolescence mediate the link between migration status and internalizing mental health problems, possibly with regard to specific contexts of measurement. Beyond the self-concept, future studies could consider the children's or their families' cultural orientations including 
the significance of religion, or perceived discrimination at school. For example, Schunck, Reiss and Razum [70] provided evidence that perceived discrimination could mediate mental health disparities between immigrant and non-immigrant adults in Germany.

\section{Implications}

Our study findings overall underlined the importance of the self-concept for the mental health of children and adolescents. Our findings moreover emphasized that a positive self-concept is especially relevant during adolescence [71]. Although our study did not directly address the practical relevance of these findings, previous intervention studies repeatedly demonstrated that a strong self-concept has positive implications for youth's mental health in school contexts. Rousseau et al. [72] evaluated a school-based prevention program that uses arts and selfexpression to strengthen the self-concept of immigrant and multi-ethnic youth. Children enrolled in this program reported higher levels of well-being in comparison to a control group. Consistent evidence for addressing students at a younger age was provided by De Bettignies and Goldstein [73]: Improvisational theatre classes in elementary schools were found to improve children's self-concept and mitigate internalizing mental health problems.

\section{Conclusions}

The proportion of immigrant children and adolescents in Germany has been increasing over recent years. Interdisciplinary practice and research integrating developmental science and clinical child psychology is required to not only consider highly diverse backgrounds among children and adolescents but also needs to acknowledge the fact that those from immigrant backgrounds have higher symptom levels of depression and anxiety. While our study supported such mental health disparities between immigrant and non-immigrant groups when assessed in school settings, data did not support that lower levels of the self-concept mediate these associations. Beyond replication studies to substantiate our findings, the search for underlying mediators needs to be continued with more specific research designs and additional focus on other migration-related candidate variables.

\section{Abbreviations \\ BAI-Y: Beck Anxiety Inventory for Youth; BDI-Y: Beck Depression Inventory for Youth; BSCI-Y: Beck Self-Concept Inventory for Youth; BYI-II: Beck Youth Inven- tory Second Edition; Cls: Confidence intervals; ISCED: International Standard Classification of Education; SES: Socio-economic status.}

\section{Acknowledgements}

The investigators gratefully thank research assistants, Eva Krey, Jasmine Golembe and Cordula Behr, for their contributions to the research project, schools and communities who were key to recruitment in this study and, finally, participating children and adolescents for contributing their time and effort to support our research.

\section{Authors' contributions}

JB contributed to the study by conceptualization, methodology, data interpretation, study coordination and by drafting and revising parts of the article. CC contributed to the study by conceptualization, methodology, data curation, data interpretation and by drafting parts of the article. SS contributed to the study by investigation, conceptualization and providing resources. GS contributed to the study by supervising the research project, funding acquisition, providing resources, revising drafts of the manuscript. All authors provided critical feedback on the manuscript and gave their approval for publication.

\section{Funding}

Open Access funding enabled and organized by Projekt DEAL. Data collection of this study was supported by "Pearson Assessment \& Information GmbH". Publication of the study was supported by the DFG Open Access Publication Funds of the Ruhr-University Bochum. The funders had no active role in study design, data collection and analysis, interpretation of data, or preparation of the manuscript.

\section{Availability of data and materials}

The dataset supporting the conclusions of this article is available from the corresponding author and GS on reasonable request. Measures used for the primary outcomes can be obtained from the "Pearson Assessment \& Information $\mathrm{GmbH}^{\prime \prime}$

\section{Declarations}

\section{Ethics approval and consent to participate}

This study was conducted in accordance with the ethical guidelines of the Germany Psychological Society. All participating children and their parents gave written informed consent in accordance with the Declaration of Helsinki. The full study protocol was approved by the Ethics Committee of the Faculty of Psychology at Ruhr-University Bochum (2016-122).

\section{Consent for publication}

Not applicable.

\section{Competing interests}

The authors declare that they have no competing interests.

\section{Author details}

${ }^{1}$ Faculty of Psychology, Department of Child and Family Research, RuhrUniversity, Bochum, Germany. ${ }^{2}$ Faculty of Psychology, Department of Clinical Child and Adolescent Psychology, Ruhr-University, Bochum, Germany. ${ }^{3}$ University Children's Hospital, Ruhr-University, Bochum, Germany.

Received: 9 October 2020 Accepted: 22 March 2021

Published online: 23 April 2021

\section{References}

1. Beck JS, Beck AT, Jolly JB. Beck youth inventories. 2nd ed. San Antonio: Psychological Corporation; 2005. p. 42.

2. Groen G, Petermann F. Depressive Kinder und Jugendliche. Berlin: Hogrefe; 2011.

3. Ravens-Sieberer U, Klasen F, Petermann F. Psychische Kindergesundheit. Kind und Entw. 2016;25:4-9. https://doi.org/10.1026/0942-5403/a000183.

4. Pine DS, Cohen P, Gurley D, Brook J, Ma Y. The risk for early-adulthood anxiety and depressive disorders in adolescents with anxiety and depressive disorders. Arch Gen Psychiatry. 1998;55:56-64. https://doi.org/10. 1001/archpsyc.55.1.56.

5. Carta MG, Bernal M, Hardoy MC, Haro-Abad JM. Migration and mental health in Europe (the state of the mental health in Europe working group: appendix 1). Clin Pract Epidemiol Ment Health. 2005;1:13. https:// doi.org/10.1186/1745-0179-1-13.

6. Belhadj Kouider E, Koglin U, Petermann F. Emotional and behavioral problems in migrant children and adolescents in Europe: a systematic 
review. J Clin Child Adolesc Psychol. 2014;23:373-91. https://doi.org/10. 1007/s00787-013-0485-8.

7. Lustig SL, Kia-Keating M, Knight WG, Geltman P, Ellis H, Kinzie JD, Saxe GN. Review of child and adolescent refugee mental health. J Am Acad Child Adolesc Psychiatry. 2004;43:24-36. https://doi.org/10.1097/00004583200401000-00012.

8. Murad S, Joung I, Lenthe FJ, Bengi-Arslan L, Crijnen A. Predictors of self-reported problem behaviours in Turkish immigrant and Dutch adolescents in the Netherlands. J Child Psychol Psychiatry. 2003;44:412-23. https://doi.org/10.1111/1469-7610.00131.

9. Plener PL, Munz L, Allroggen M, Kapusta ND, Fegert JM, Groschwitz RC. Immigration as risk factor for non-suicidal self-injury and suicide attempts in adolescents in Germany. Child Adolesc Psychiatry Ment Health. 2015;9:34. https://doi.org/10.1186/s13034-015-0065-4.

10. Van Oort FV, van der Ende J, Crijnen AA, Verhulst FC, Mackenbach JP, Joung IM. Ethnic disparities in problem behaviour in adolescence contribute to ethnic disparities in social class in adulthood. Soc Psychiatry Psychiatr Epidemiol. 2007:42:50-6.

11. Frank L, Yesil-Jürgens R, Born S, Hoffmann R, Santos-Hövener C, Lampert T. Maßnahmen zur verbesserten Einbindung und Beteiligung von Kindern und Jugendlichen mit Migrationshintergrund in KiGGS Welle 2. Journal of Health Monitoring. 2018;3:134-51. https://doi.org/10.17886/ RKI-GBE-2018-017.

12. Stevens GWJM, Vollebergh AM. Mental health in migrant children. J Child Psychol Psychiatry. 2008;49:276-94. https://doi.org/10.1111/j.1469-7610. 2007.01848.x.

13. Szapocznik J, Kurtines WM. Family psychology and cultural diversity. Opportunities for theory, research and application. Am Psychol. 1993;48:400-7. https://doi.org/10.1037/0003-066X.48.4.400.

14. Sam DL, Virta E. Intergenerational value discrepancies in immigrant and host-national families and their impact on psychological adaptation. J Adolesc. 2003;26:213-31. https://doi.org/10.1016/s0140-1971(02)00129-X.

15. Zwirs BWC, Burger H, Buitelaar JK, Schulpen TWJ. Ethnic differences in parental detection of externalizing disorders. Eur Child Adolesc Psychiatry. 2006;15:418-26. https://doi.org/10.1007/s10802-007-9112-9.

16. Zwirs BWC, Burger H, Schulpen TWJ, Buitelaar JK. Different treatment thresholds in non-Western children with behavioral problems. J Am Acad Child Adolesc Psychiatry. 2006;45(4):476-83. https://doi.org/10.1097/01. chi.0000192251.46023.5a.

17. Park SY, Lee H, Kang M. Factors affecting health literacy among immigrants: systematic review. Eur J Public Health. 2018;28:406-7.

18. Babiker I, Cox J, Miller P. The measurement of cultural distance and its relationship to medical consultations: symptomatology and examination performance of overseas students at Edinburgh University. Int J Soc Psychiatry. 1980;15:109-16. https://doi.org/10.1007/BF00578141.

19. Kashima ES, Abu-Rayya HM. Longitudinal associations of cultural distance with psychological well-being among Australian immigrants from 49 countries. J Cross Cult Psychol. 2014;45:587-600. https://doi.org/10.1177/ 0022022113519857

20. Gutmann M, Aysel M, Özlü-Erkilic Z, Popow C, Akkaya-Kalaycic T. Mental health problems of children and adolescents, with and without migration background, living in Vienna. Austria Child Adolesc Psychiatry Ment Health. 2019;13:35. https://doi.org/10.1186/s13034-019-0295-y.

21. Trautwein U, Köller O, Lüdtke O, Baumert J. Self-esteem, academic selfconcept, and achievement: how the learning environment moderates the dynamics of the self-concept. J Pers Soc Psychol. 2006;90:334-9. https://doi.org/10.1037/0022-3514.90.2.334.

22. Jekauc D, Wagner MO, Herrmann C, Hegazy K, Woll A. Does physical selfconcept mediate the relationship between motor abilities and physical activity in adolescents and young adults? PLoS ONE. 2017;12:1. https:// doi.org/10.1371/journal.pone.0168539.

23. Thomsen T, Lessing N, Greve W, Dresbach S. Selbstkonzept und Selbstwert. In: Lohaus A, editor. Entwicklung des Jugendalters. Berlin: Springer; 2018. p. 91-111.

24. Showers CJ, Zeigler-Hill V. Pathways among self-knowledge and selfesteem: How are self-esteem and self-knowledge linked? Are these links direct or indirect? In: Kernis MH, editor. Self-esteem issues and answers: a source book of current perspectives. New York: Psychology Press; 2006. p. 216-23. https://doi.org/10.1177/0146167206294872.
25. Showers CJ, Ditzfeld CP, Zeigler-Hill V. Self-concept structure and the quality of self-knowledge. J Pers. 2015;83(5):535-51. https://doi.org/10. 1111/jopy. 12130.

26. Crocetti E, Rubini M, Luyckx K, Meeus W. Identity formation in early and middle adolescents from various ethnic groups: From three dimensions to five statuses. J Youth Adolesc. 2008;37:983-96. https://doi.org/10.1007/ s10964-007-9222-2.

27. Möller, J \& Trautwein, U. Selbstkonzept. In: Pädagogische Psychologie. Heidelberg: Springer, 2009.

28. Emler N. Self esteem: the costs and causes of low self worth. New York: Joseph Rowntree Foundation, York Publishing Services Ltd; 2001.

29. Erkolahti R, llonen T. Saarijärvi S \& Terho P Self-image and depressive symptoms among adolescents in a non-clinical sample. Nord J Psychiatry. 2003;57:447-51. https://doi.org/10.1080/08039480310003461.

30. Räty LKA, Larsson G, Söderfelt BA, Wilde Larsson BH. Psychosocial aspects of health in adolescence: the influence of gender, and general selfconcept. J Adolesc Health. 2005;36:521-8. https://doi.org/10.1016/j.jadoh ealth.2004.10.006.

31. Martinsen KD, Neumer S-P, Holen S, Waaktaar T, Sund AM, Kendall PC. Self-reported quality of life and self-esteem in sad and anxious school children. BMC Psychol. 2016;4(1):45. https://doi.org/10.1186/ s40359-016-0153-0.

32. Roebers $C M$, Schneider W. Self-concept and anxiety in immigrant children. Int J Beh Dev. 1999;23:125-47.

33. Bourque F, van der Ven E, Malla A. A meta-analysis of the risk for psychotic disorders among first- and second-generation immigrants. Psychol Med. 2011:41:897-910. https://doi.org/10.1017/S0033291710001406.

34. Ceri V, Özlü-Erkilic Z, Özer Ü, KadakT, Winkler D, Dogangün B, AkkayaKalayci T. Mental health problems of second generation children and adolescents with migration background. Int J Psychiatry Clin Pract. 2017:2:142-7. https://doi.org/10.1080/13651501.2017.1291820.

35. Kizilhan Jl. Potenziale und Belastungen psychosozialer Netzwerke in der Migration. In: Borde T, Davis M, editors. Migration und seelische Gesundheit, psychische Belastungen und Potenziale. Frankfurt: Mabuse; 2007. p. 53-68.

36. Odag-Wieacker I. Schwierigkeiten in der Identitätsbildung bei Kindern und Jugendlichen in Folge der Migration ihrer Elterngeneration. In: Burkhart-Mußmann C, Dammach B, editors. Migration, Flucht \& Kindesentwicklung. Frankfurt: Brandes und Apsel; 2016.

37. Uslucan HH. Lebensweltliche Verunsicherung türkischer Migranten. Psychosozial. 2005;28:111-22.

38. Cole DA, Maxwell SE, Martin JM, Peeke LG, Seroczynski AD, Tram JM, Hoffmann KB, Ruiz MD, Jaquez F, Maschman T. The development of multiple domains of child and adolescent self-concept: a cohort sequential longitudinal design. Child Dev. 2001;72:1723-46. https://doi.org/10.1111/ 1467-8624.00375.

39. Meeus W. The study of adolescent identity formation 2000-2010: A review of longitudinal research. J Res Adolesc. 2011;21:75-94. https://doi. org/10.1111/j.1532-7795.2010.00716.x.

40. Foulkes $L$, Blakemore $S$. Is there heightened sensitivity to social reward in adolescence? Curr Opin Neurol. 2016;40:81-5. https://doi.org/10.1016/j. conb.2016.06.016.

41. Sherman LE, Payton AA, Hernandez LM, Greenfield PM, Dapretto M. The power of the like in adolescence: effects of peer influence on neural and behavioral responses to social media. Psychol Sci. 2016;27:1027-35. https://doi.org/10.1177/0956797616645673.

42. Siefen RG. Psychische Entwicklungsrisiken bei Kindern und Jugendlichen mit Migrationshintergrund. In: Borde T, David M, editors. Kinder und Jugendliche mit Migrationshintergrund. Frankfurt: Mabuse; 2015 p. 107-19.

43. Schepker R. Jugendliche in der Migration. In: Machleidt W, Kluge U, Sieberer M, Heinz A, editors. Praxis der interkulturellen Psychiatrie und Psychotherapie. München: Elsevier; 2018.

44. Mitchell N. Academic achievement among caribbean immigrant adolescents: the impact of generational status on academic self-concept. Prof Sch Couns. 2005:8(3):209-18.

45. Marsh HW, Parada RH, Ayotte V. A Multidimensional perspective of relations between self-concept (Self-Description Questionnaire II) and adolescent mental health (Youth Self-Report). Psychol Assess. 2004;16:27-41. https://doi.org/10.1037/1040-3590.16.1.27. 
46. Ybrandt $\mathrm{H}$. The relation between self-concept and social functioning in adolescence. J Adolesc. 2008;31:1-16. https://doi.org/10.1016/j.adole scence.2007.03.004.

47. Coelho VA, Marchante M, Jimerson SR. Promoting a positive middle school transition: a randomized-controlled treatment study examining self-concept and self-esteem. J Youth Adolesc. 2017;46:558-69. https:// doi.org/10.1007/s10964-016-0510-6.

48. Ganzeboom HBG, De Graaf PM, Treiman DJ. A standard international socio-economic index of occupational status. Soc Sci Res. 1992;21:156. https://doi.org/10.1016/0049-089X(92)90017-B.

49. Siefen RG, Busch J. Beck youth inventories. 2nd ed. Frankfurt: Pearson Assessment; 2018.

50. American Psychiatric Association. Diagnostic and statistical manual of mental disorders (4th ed., Text Revision). Washington, DC: APA, 2000.

51. IBM Corp. IBM SPSS Statistics for Mac, Version 25.0. Armonk, NY: IBM Corp., 2017.

52. R Core Team. R: A language and environment for statistical computing. R Foundation for Statistical Computing. Wien, Österreich: 2017. https:// www.R-project.org/.

53. Hemmerich, W. StatistikGuru: Rechner zur Adjustierung des a-Niveaus. 2016. Retrieved from https://statistikguru.de/rechner/adjustierungdes-alphaniveaus.html Accessed 29 Jan 2021

54. Holm S. A simple sequentially rejective multiple test procedure. Scand J Stat. 1979;6:65-70.

55. Edwards JR, Lambert LS. Methods for integrating moderation and mediation: a general analytical framework using moderated path analysis. Psychol Methods. 2007;12:1-22. https://doi.org/10.1037/1082989X.12.1.1.

56. Hayes AF. Introduction to mediation, moderation, and conditional process analysis. 2nd Edition. A regression-based approach. New York: Guilford Press; 2018.

57. Baron RM, Kenny DA. The moderator-mediator variable distinction in social psychological research: conceptual, strategic, and statistical considerations. JPSP. 1986;51:1173-82. https://doi.org/10.1016/00221031(82)90046-4.

58. Sobel ME. Asymptotic confidence intervals for indirect effects in structural equation models. Sociol Methods Res. 1982;13:290-312. https:// doi.org/10.2307/270723

59. Coll CG, Marks AK, editors. The immigrant paradox in children and adolescents: is becoming American a developmental risk? Washington: American Psychological Association; 2012.

60. Vaage AB, Tingvold L, Hauff E, Van Ta T, Wentzel-Larsen T, Clench-Aas J, Thomsen PH. Better mental health in children of Vietnamese refugees compared with their Norwegian peers: a matter of cultural difference? Child Adol Psychiatry Ment Health. 2009;3:34. https://doi.org/10.1186/ 1753-2000-3-34.

61. Markides K, Rote S. Immigrant health paradox. In: Scott RA, Kosslyn S, editors. Emerging trends in social and behavioral sciences. Hoboken: Wiley; 2015. p. 1-15.

62. Mood C, Jonsson JO, Låftman SB. Immigrant integration and youth mental health in four European countries. Eur Sociol Rev. 2016;32:71629. https://doi.org/10.1093/ESR/JCW027.
63. Dimitrova R, Chasiotis A, van de Vijver F. Adjustment outcomes of immigrant children and youth in Europe: a meta-analysis. Eur Psychol. 2016;21:150-62. https://doi.org/10.1027/1016-9040/a000246.

64. Brettschneider A, Hölling H, Schlack R, Ellert U. Psychische Gesundheit von Jugendlichen in Deutschland. Bundesgesundheitsblatt Gesundheitsforschung Gesundheitsschutz. 2015;58:474-89.

65. Marks AK, Ejesi K, García CC. Understanding the US immigrant paradox in childhood and adolescence. Child Dev Perspect. 2014;8:59-64. https:// doi.org/10.1111/cdep.12071.

66. Krannich M, Goetz T, Lipnevich AA, Bieg M, Roos A, Becker ES, Morger $V$. Being over- or underchallenged in class: effects on students' career aspirations via academic self-concept and boredom. Learn Individ Differ. 2019;69:206-18. https://doi.org/10.1016/j.lindif.2018.10.004.

67. Houck G, Kendall J, Miller A, Morrell P, Wiebe G. Self concept in children and adolescents with ADHD. Pediatr Nurs. 2011;26:239-47. https://doi. org/10.1016/j.pedn.2010.02.004.

68. Marsh HW, Ellis LA, Craven RG. How do preschool children feel about themselves? Unraveling measurement and multidimensional selfconcept structure. Dev Psychol. 2002;38:376-93. https://doi.org/10.1037/ 0012-1649.38.3.376

69. Marsh HW, Parada RH, Yeung AS, Healey J. Aggressive school troublemakers and victims: a longitudinal model examining the pivotal role of self-concept. J Educ Psychol. 2001;93:411-9. https://doi.org/10.1037// 0022-0663.93.2.411.

70. Schunck R, Reiss K, Razum O. Pathways between perceived discrimination and health among immigrants: evidence from a large national panel survey in Germany. Ethn Health. 2014;20:493-510. https://doi.org/10. 1080/13557858.2014.932756.

71. Schulte-Körne G. Mental health problems in a school setting in children and adolescents. Dtsch Arztebl Int. 2016;113(11):183-90. https://doi.org/ 10.3238/arztebl.2016.0183.

72. Rousseau C, Drapeau A, Lacroix L, Bagilishya D, Heusch N. Evaluation of a classroom program of creative expression workshops for refugee and immigrant children. J Child Psychol Psychiatry. 2005;46(2):180-5. https:// doi.org/10.1111/j.1469-7610.2004.00344.x.

73. De Bettignies BH \& Goldstein TR. Improvisational theater classes improve self-concept. Psychology of Aesthetics, Creativity, and the Arts. 2019. https://psycnet.apa.org/record/2019-29428-001. Accessed 31 Jan 2020.

74. UNESCO Institute of Statistics. International Standard Classification of Education ISCED 2011. 2012. http://uis.unesco.org/sites/default/files/ documents/international-standard-classification-of-education-isced2011-en.pdf. Accessed 31 Jan 2020.

\section{Publisher's Note}

Springer Nature remains neutral with regard to jurisdictional claims in published maps and institutional affiliations.

Ready to submit your research? Choose BMC and benefit from:

- fast, convenient online submission

- thorough peer review by experienced researchers in your field

- rapid publication on acceptance

- support for research data, including large and complex data types

- gold Open Access which fosters wider collaboration and increased citations

- maximum visibility for your research: over $100 \mathrm{M}$ website views per year

At BMC, research is always in progress.

Learn more biomedcentral.com/submissions 